\title{
Association of Serum Phosphate and Related Factors in ESRD-Related Vascular Calcification
}

\author{
Cai-Mei Zheng, ${ }^{1}$ Kuo-Cheng Lu, ${ }^{2}$ Chia-Chao Wu, ${ }^{3,4}$ Yung-Ho Hsu, ${ }^{1}$ and Yuh-Feng Lin ${ }^{1}$ \\ ${ }^{1}$ Division of Nephrology, Department of Medicine, Taipei Medical University-Shuang Ho Hospital, \\ Taipei Medical University, Taipei 235, Taiwan \\ ${ }^{2}$ Department of Medicine, Cardinal Tien Hospital, School of Medicine, Fu Jen Catholic University, Taipei 231, Taiwan \\ ${ }^{3}$ Division of Nephrology, Department of Medicine, Tri-Service General Hospital, National Defense Medical Center, Taipei 114, Taiwan \\ ${ }^{4}$ Graduate Institute of Microbiology and Immunology, National Defense Medical Center, Taipei 114, Taiwan
}

Correspondence should be addressed to Yuh-Feng Lin, linyf@shh.org.tw

Received 11 March 2011; Accepted 12 March 2011

Academic Editor: Biagio Raffaele Di Iorio

Copyright (c) 2011 Cai-Mei Zheng et al. This is an open access article distributed under the Creative Commons Attribution License, which permits unrestricted use, distribution, and reproduction in any medium, provided the original work is properly cited.

\begin{abstract}
Vascular calcification is common in ESRD patients and is important in increasing mortality from cardiovascular complications in these patients. Hyperphosphatemia related to chronic kidney disease is increasingly known as major stimulus for vascular calcification. Hyperphosphatemia and vascular calcification become popular discussion among nephrologist environment more than five decades, and many researches have been evolved. Risk factors for calcification are nowadays focused for the therapeutic prevention of vascular calcification with the hope of reducing cardiovascular complications.
\end{abstract}

\section{Introduction}

Vascular calcification is a kind of extraosseous calcification and is associated with aging physiologically, and a number of disorders including ESRD, diabetes mellitus, and cardiovascular disease pathologically. Multifactorial processes contribute to $\mathrm{VC}$ in which derangements in calcium and phosphorus homeostasis plays an important role and becomes popular therapeutic target nowadays. In ESRD patients with vascular calcification, a mixture of intimal and medial calcification has been observed in the effected vessels with dominant medial involvement. The risk of CVD mortality in ESRD patients with vascular calcification is 20 to 30 times higher than that of the general population [1-5].

Although phosphate is important for diverse cellular and physiological functions, impaired renal function with resultant phosphate accumulation with consequent bone and mineral disorders and vascular calcification are major problems among nephrologists. The increased risk of CVD mortality by hyperphosphatemia was partially explained by the predisposition of this population to vascular calcification [6-8]. (Figure 1) Even in early stage CKD, serum phosphorus level disturbances are proved to promote vascular calcification, hypertension, myocardial hypertrophy, and heart fai- lure [9-11]. Current understanding of relationship between phosphorus and those disorders becomes popular in medical field, with the hope of halting or retarding the vascular calcification from the very early status in those patients.

\section{Traditional Concepts in ESRD Patients with Vascular Calcification}

2.1. Vascular Calcification in ESRD Patients. Vascular calcification (VC), an extra osseous calcification of arteries, is strongly associated with CKD patients with or without hemodialysis. Two types of VC include neointimal calcification, which occurs in large and medium-sized arteries, and medial calcification, which occurs in arteries of any size, including arterioles. $\mathrm{VC}$ is an important indicator of atherosclerosis, and its occurance directly predict prognosis of atherosclerotic disease [12]. Uremic atherosclerotic plaques are more calcified and fibroatheromatous than those in aging, with similar cellular infiltrates [13] and more of tunica media involvement $[14,15]$. VC in ESRD patients, those especially found in the tunica media of large arteries, may lead to increased stiffening and decreased compliance of these vessels. Consequent increased arterial pulse wave velocity, pulse pressure, and impair arterial dispensability result 


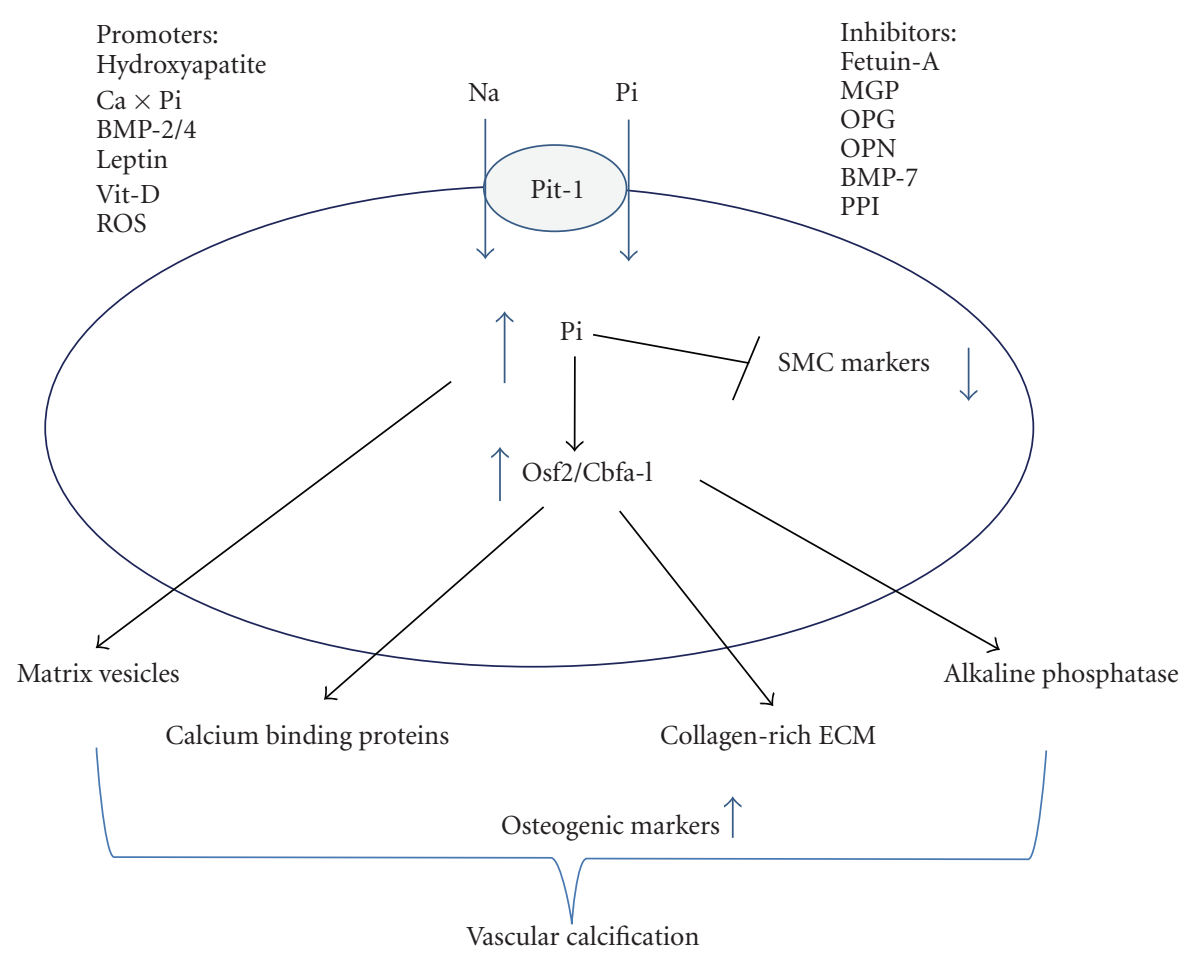

FIGURE 1: Mechanisms of VSMC osteogenesis during vascular calcification in chronic kidney disease. VSMC upregulate expression of transcription factors Osf2/Cbfal which were enhanced by ROS, leptin, vitamin D, increased CaxP product, or high $\mathrm{PO}_{4}$ (Pi) levels induced by Pit-1. VSMC activation occurs in part as a result of the phenotypic switch of VSMCs into osteoblast-like cells. VSMCs that have acquired an osteogenic phenotype express ALP and produce hydroxyapatite crystals. Calcification inhibitors such as PPi inhibit hydroxyapatite precipitation, whereas fetuin-A, MGP, OPG, OPN, and BMP-7 antagonize calcification. VSMC: Vascular smooth muscle cells, Osf2/Cbfa1: Osteoblast-specific transcription factor, ROS: Reactive oxygen species, CaxP product: Calciumx phosphate produce, $\mathrm{PO} 4(\mathrm{Pi})$ : $\mathrm{Phosphate,} \mathrm{Pit-}$ 1: Sodium-phosphate cotransporter-1, ALP: Alkaline phosphatase, PPi: Pyrophosphate, MGP: Matrix Gla protein, OPG: Osteoprotegerin, OPN: Osteopontin, BMP: Bone morphogenic protein.

in increase afterload and left ventricular hypertrophy, which finally compromise coronary perfusion with development of congestive heart failure $[7,10,16-19]$.

\subsection{Risk Factors of Vascular Calcification in ESRD Patients.} Risk factors for premature VC in ESRD patients are different from the traditional atherogenic risk factors. Hyperparathyroidism and alteration in Ca-P mineral metabolism, especially hyperphosphatemia, modulate renal osteodystrophy and vascular medial calcification [20, 21]. Microinflammation with chronically elevated acute phase protein CRP relates with intimal calcification and predicts the CV mortality. The presence of $\mathrm{C}$. pneumoniae in arterial walls and atherosclerotic lesions also related this persistant infection with atherosclerotic vascular lesions in CKD with or without dialysis patients $[13,19]$. Longer hemodialysis duration is also found to be significantly relate with severe vascular calcification $[4,5,21,22]$. Furthermore, hyperglycemia and hyperphosphatemia are two most significant factors to be considered in ESRD patients with and without diabetes mellitus, respectively [23].

2.3. Role of Phosphate in ESRD with Vascular Calcification. Serum phosphate concentration is usually maintained within
2.5 to $4.5 \mathrm{mg} / \mathrm{dL}$ by a variety of mechanisms until renal disease has progressed to approximately CKD stage 5 or ESRD $[24,25]$. Adaptation of nephrons in attempt to preserve phosphate homeostasis in ESRD patients plays an important role for VC. Hyperphosphatemia result in secondary hyperparathyroidism, calcium and vitamin $\mathrm{D}$ derangements, vascular calcification, and mineral bone disorders. Additionally, hyperparathyroid state and altered vitamin D status in ESRD patients also play a major role in extraosseous calcifications [1, 26-28]. Higher serum phosphorus levels may increase serum PTH levels even in healthy individuals [29]. Our previous study revealed serum PTH levels may stimulate inflammatory marker IL-6 production in HD patients [30, 31]. Higher levels of serum IL-6 and hsCRP also associated with increased VC and CVD risk. Higher serum phosphorus levels also inhibit 1,25-dihydroxyvitamin D synthesis [32, 33]. Lower 1,25-dihydroxyvitamin $\mathrm{D}$ status was associated with myocardial dysfunction $[27,34]$ and increased coronary vascular calcification $[26,27,35]$. The effects of vitamin D on vascular calcification are biphasic pattern with both excess, and deficiency may promote its development [17].

2.4. Mechanism of Vascular Calcification. Vascular calcification involves two distinct events, smooth muscle cell 
transformation and mineralization. Normally, blood vessels express inhibitors of mineralization, like pyrophosphate, matrix Gla protein, fetuin A, and loss of these inhibitors may result in spontaneous vascular calcification. Fetuin A also plays a role in inhibition of CaxP precipitation [36].

The presence of bone-like tissue including osteoblastlike cells and hematopoietic elements within atherosclerotic plaque suggest $\mathrm{VC}$ as osteogenic differentiation of vessel wall cells [37-40]. A signal from AS plaque induces expression of potent osteogenic differentiation factor BMP-2a, with bone matrix and calcium hydroxyapatite crystals deposition in the arterial wall cells $[41,42]$. Osteoprotegerin (OPG) $[43,44]$ inactivates the osteoclasts by blocking RANK activation. In dialysis patients, serum OPG levels are associated and predict progressive vascular calcification especially when CRP level is increased. Unregulated, degenerative calcification process within advanced AS plaque is progressive and severe in ESRD patients.

Hyperphosphatemia plays a very important role in ESRD related arteropathy $[6,7,45]$. In hyperphosphatemic environment $(\mathrm{P}>2.4 \mathrm{mM})$, the vascular smooth muscle cell culture systems revealed both osteochondrogenic phenotypic change and mineralization through sodium-dependent phosphate cotransporter, Pit-1 [39, 46, 47]. There was loss of smooth muscle-specific gene expression and upregulartion of bone differentiation genes that translate into differential factors (Osteocalcin, osteopontin and Runx2, etc.), with the resultant osteogenic differentiation. Calcium deposition also occurs in prolonged hypercalcemic $(>2.6 \mathrm{mM})$ environment. Calcium increases Pit-1 mRNA levels, increases smooth muscle cell sensitization to phosphorus, and results in osteoblastic differentiation. Elevated serum calcium was also associated with elevated CaxP product, altered alkaline phosphatase, and decreased marix Gla protein, which together play a role in VC [48].

Recent study revealed that calcium phosphate deposition may trigger the osteogenic changes. Calcium phosphate deposition (CPD) occurs as a cellular independent phenomenon which depends on calcium, phosphate, and hydroxyl ions concentration, but not on CaxPi concentration products [49]. A mouse lacking the VC inhibitor matrix-Gla protein had spontaneous extensive extraosseous calcifications despite normal $\mathrm{Ca}$ and $\mathrm{P}$ concentrations [50]. Mineralization is actively inhibited and prevented in the arteries. The loss of calcification inhibitors and CPD may lead to specific osteogene expression and VSMC differentiation and finally result in vascular calcification [49].

A genetic mechanism of hyperphosphatemia on vascular calcification was cited by Wu-Wong et al. in 2007. Elevated $\mathrm{P}$ modulates through VDR mRNA stability and PPAR $\gamma$ mediated gene expression may lead to its detrimental effects including vascular calcification [20].

The calcium-sensing receptor (CaSR), which is expressed in vascular wall, plays key role in inhibition of vascular calcification in patients with chronic kidney disease (CKD). After CaSR stimulation, many intracellular signaling events occur via MEK1/ERK1,2 and PLC pathways and lead to proliferation of vascular smooth muscle cells. CaSR-mediated PLC activation is important for SMC survival and protection against apoptosis [51, 52]. Calcimimetic agents act on CaSR increasingly known nowadays for treatment of SHPT and CKD-MBD. Calcimimetics activate vascular calciumsensing receptor and modulate the expression of VC inhibitor proteins like matrix Gla [53]. Calcimimetics were shown to have better plasma PTH controlling than calcitriol in uremic rats [54]. It also revealed that combination of Calcimimetics with calcitriol prevents VC and reduced mortality. Apart from controlling serum PTH levels, these agents also reduce calcium $(\mathrm{Ca})$, phosphate $(\mathrm{P})$, and $\mathrm{CaxP}$ product levels, which is more beneficial than other calcium containing phosphate binders and vitamin $\mathrm{D}$ analogues $[53,54]$. These studies revealed that those agents may even reverse some vascular abnormalities in CKD.

\section{Contemporary Concepts in ESRD Patients with Vascular Calcification}

3.1. Role of Inflammation and Oxidative Stress. HD patients with secondary hyperparathyroidism may be under an environment of increased serum inflammatory cytokines and oxidative stress $[30,55]$. High PTH levels, inflammation, and oxidative stress may involve together in CKDMBD and cardiovascular calcification in SHP patients. Our recent study showed calcitriol can effectively suppress PTH secretion and reduce inflammatory markers including CRP and IL-6 [31]. While using vitamin D analogues in CKD patients, we have to note that, on one side, with increasing survival benefit, and on the other side, with some risks of vascular calcification. Thus, vitamin D in its form of less hypercalcemic agent paricalcitol, combination therapy with calcimimetic agents is becoming popular nowadays. Zhao et al. [56] demonstrate recently that increased mitochondrial membrane potential may lead to intracellular and mitochondrial reactive oxygen species (ROS) stimulation, with resultant superoxide production, and increased oxidative stress. These ROS mediate p65 nuclear translocation which is found to associate with phosphate-induced VC [56]. Osteogenic differentiation factor bone morphogenic protein2a (BMP-2a), a well-known mediator of VC, is nowadays found to be a molecular link between oxidative stress and arterial stiffness due to vascular calcification $[41,57]$.

3.2. FGF23-Klotho Axis. The fibroblast growth factor-23 (FGF-23) and klotho genes are growingly known to regulate phosphate homeostasis recently [58]. Fibroblast growth factor-23 inhibits proximal renal and intestinal phosphorus absorption and klotho gene express in renal distal convoluted tubules and parathyroid gland. In mice, deletions of klotho and FGF-23 genes found to result in hyperphosphaemia, vascular calcification, arteriosclerosis, elevated $1,25(\mathrm{OH})_{2} \mathrm{D}$, and osteopenia.

Patients with CKD have elevated FGF-23 levels early in the course of disease before clinically significant hyperphosphatemia occurs. Increased circulating FGF-23 target the remnant nephrons, enhance phosphate excretion, and inhibit $1,25(\mathrm{OH})_{2} \mathrm{D}_{3}$ production. Thus, frank hyperphosphatemia does not develop until GFR $<30 \mathrm{~mL} / \mathrm{min}$. FGF-23 concentrations directly correlate with renal function. Higher 
FGF-23 levels indicate rapidly progressive CKD, and also predict mortality in HD patient, thus, FGF-23 level may become important as creatinine level in kidney function assessment in future [59]. FGF-23 in earlier CKD increase phosphate excretion and prevent the development of vascular calcification. Higher FGF-23 also lead to reduced serum levels of $1,25(\mathrm{OH})_{2} \mathrm{D}$ in early CKD despite high PTH. Its phosphaturic and hypovitaminosis $\mathrm{D}$ effects are more important than PTH in earlier stages of CKD. The resultant low serum $1,25(\mathrm{OH})_{2} \mathrm{D}$ and hypocalcemia enhance PTH secretion and progress to secondary hyperparathyroidism. FGF-23, together with cofactor klotho, act on FGF receptor in parathyroid gland and reduce its secretion. But, in later stages of CKD, FGF23 resistance and hyperphosphatemia lead to secondary HPTH despite high FGF23 levels. Due to progressive nephron loss, the number of nephrons responds to FGF-23 reduced and phosphate retension occurs in late stage CKD. Abnormally high FGF23 levels in later stages of CKD lead to vascular dysfunction, left ventricular hypertrophy, and early mortality. Aortic calcification can be predicted by measuring FGF-23 level in hemodialysis patients [60]. Phosphate binders or use of long-acting PTH analogs to reduce intestional phosphate reabsorption in earlier stages CKD may reduce FGF-23 production and prevent those complications. In future, FGF-23 may be an important therapeutic target in management of CKD and hyperphosphatemia.

3.3. Role of Metabolic Acidosis. Metabolic acidosis which is very common in CKD patients may lead to bone dissolution by osteoclast activation and osteoblast inhibition [61, 62]. Our studies prove that acute correction of metabolic acidosis improves osteoblast function, increases $1,25(\mathrm{OH})_{2} \mathrm{D}_{3}$ levels, and attenuates circulating PTH activity in chronic renal failure patients, and it underlines the importance of maintaining normal acid-base homeostasis in chronic renal failure [6365]. Mendoza et al. [66] hypothesize an assumption that the response of extraosseous calcification to metabolic acidosis may be similar to that of bone, and, given that metabolic acidosis impairs bone mineralization, it is likely to attenuate the extraosseous calcification processes. They investigate the in vivo effect of metabolic acidosis on the development of vascular and other soft-tissue calcifications in a rodent model of uremia. Their results show that metabolic acidosis eventually prevents the development of calcitriol-induced extraskeletal calcifications in uremic rats, even with elevated plasma $\mathrm{Ca}$ and $\mathrm{P}$ levels. Metabolic acidosis plays a complex mechanism in VC, including stimulating of the solubility of Ca-P deposits, suppressing parathyroid secretion, inhibiting some osteogenic enzymes, blocking bone matrix formation, modulating the upregulation of Pit-1, and finally blocking phosphate uptake by the arterial smooth muscle cells [61].

3.4. Role of Adipocytokine Leptin. Increased BMI and abdominal obesity are well known to be related with metabolic syndrome and cardiovascular complications. Mediators released from adipose tissue are recently shown to be playing a role in promoting CVD. It has been shown in our previous study that adipocytokine leptin level is a marker for body adiposity in hemodialysis patients [67]. Studies conducted by Zeadin et al. [68] and Martin et al. [69] showed that a positive correlation between plasma leptin levels and CAC. It has been demonstrated that leptin promote osteoblastic differentiation of vascular smooth muscle cells and, thus, possibly lead to vascular calcification.

3.5. Role of Salivary Phosphate. In uremic patients, hyperphosphatemia is found to be poorly controlled even with the use of phosphate binders and dietary phosphate limitation in addition to dialysis. Savica et al. [70] found the role of salivary phosphate in worsening hyperphosphatemia in CKD patients. The salivary phosphorus ratio in dialysis patients was more than two times compared with healthy controls, and salivary phosphorus was five times higher than serum phosphorus in those patients. Thus, salivary phosphate binders could be an efficient approach in treating hyperphosphatemia in those patients.

3.6. Role of Pyrophosphate. Recent data suggest that deficient in pyrophosphate (PPi), a potent inhibitor of vascular calcification, may increase the medial vascular calcification in advanced kidney disease [71]. Levels of PPi are reduced in hemodialysis patients [72]. Hydrolysis of PPi is increased in aortas from uremic rats because of upregulation of alkaline phosphatase, providing a mechanism for vascular deficiency of PPi. Therefore, the data suggest that exogenous PPi may be useful in treating or preventing uremic vascular calcification. O'Neill and colleagues [73] show in uremic rats that systemic administration of pyrophosphate prevents or reduces uremia-related vascular calcification, without overt negative consequences for bone and without calcium pyrophosphate deposition disease. These findings prompt further research into the potential of pyrophosphate as treatment for vascular calcification in chronic kidney disease patients.

\subsection{New Concept on Measures to Halt Phosphorus-Related} Vascular Calcification. Factors that relate with vascular calcification process in CKD patients include phosphorus activation of the Pit- 1 receptor, bone morphogenic proteins 2 and 4, leptin, endogenous 1,25 dihydroxy vitamin D, vascular calcification activating factor, and measures of oxidative stress. These entities may become future targets for diagnosis and treatment since standard hydroxymethylglutaryl-CoA reductase inhibitors have been shown to be failing to attenuate the progressive $\mathrm{VC}$ in those patients.

Dietary and therapeutic phosphorus control stands out as an important and basic step of preventing progressive VC in CKD patients. Dietary phosphorus control plays an important regulatory role in mineral homeostasis. Phosphorus is absorbed throughout the intestines and via 2 separate processes, a sodium-independent paracellular pathway and sodium-dependent carrier-mediated transcellular pathway (NaP-IIb) [74]. Low dietary phosphorus level may increase that type II b Na-P cotransporters protein expression on enterocytes with increasing $\mathrm{P}$ absorption. The percentage of dietary absorption varies with intake. At high levels of 
intake ( $>10 \mathrm{mg} / \mathrm{kg} /$ day), approximately $70 \%$ of the ingested $\mathrm{P}$ is absorbed, whereas at lower levels of intake, as much as 80\%-90\% may be absorbed. Net intestional P absorption may be somewhat lowered in patients with ESRD. Inorganic phosphate is an important $\mathrm{P}$ which is found mostly in food additives like enhanced meat products, cereal and snack bars, flavored waters, and frozen meals, with $90 \%$ to $100 \%$ absorption. Thus, the principle of Pi management in ESRD patients is to restrict dietary Pi intake to $1 \sim 1.4 \mathrm{~g} /$ day, usage of phosphate binders as needed, and if still noncompliant can increase the dialysis time (Nocturnal HD) and frequency (Short Daily HD) with target levels of $\mathrm{Pi}<4.6 \mathrm{mg} / \mathrm{dL}$ in CKD stage 3,4 and 3.5-5.5 mg/dL in CKD stage 5 .

With concept of insufficient phosphorus removal by conventional hemodialysis, phosphate binders are taking to reduce gastrointestional phosphate absorption by virtually all hemodialysis patients. Due to potential risk of VC with calcium overload, Calcium-based phosphate binders are nowadays replaced by non-Ca-based ones. Sevelamer $\mathrm{HCl}$ [75-77] and Lanthanum carbonate [78-80] are the non-Cabased phosphate binders well known nowadays with beneficial effects on vascular calcification. Sevelamer found to exert many benefits on VC by increasing fetuin-A levels, reducing systemic inflammation and modulating lipid profiles [8183]. Efficacy and safety of new non-calcium, iron-based phosphate binder SBR759 was studied by Block et al. in a phase I clinical trial. Risks of iron accumulation and hypocalcemia were addressed but appear to be well tolerated [84].

Pyrophosphate, serve as an endogenous inhibitor of calcification and prevent hydroxyapatite formation in the vessel walls [85-87]. Pyrophosphate stands important defense against calcification in vessels without adverse effects on bone [73]. Thus, pyrophosphate becomes recent popular medication research in progress. Nitrogen-containing bisphosphonates (NCBPs) (e.g., ibandronate, alendronate, risedronate, and zoledronate) may inhibit the cardiovascular calcification $[88,89]$. Etidronate may also reduce chronic inflammatory response and decrease OPG concentrations with the resultant decrease in vascular calcification in dialysis patients [90].

Niacin lowers serum phosphate and increases HDL cholesterol in dialysis patients. Niaspan (prolonged-release nicotinic acid) lowers serum phosphate and increases HDL cholesterol in dialysis patients [91, 92]. Patients with ESRD often have markedly elevated salivary phosphate concentration, independent of food content. A recent study on thirteen HD patients with serum phosphate levels $>6.0 \mathrm{mg} / \mathrm{dL}$ chewed $20 \mathrm{mg}$ of chitosan-loaded chewing gum twice daily for $2 \mathrm{wk}$ at fast in addition to oral compound found to be a useful approach for improving treatment of hyperphosphatemia in HD patients [93]. An experiment on ammonium chloride on uremic rats having a high phosphorus diet and calcitriol treatment may prevent vascular calcification [66]. This explains that the alkaline $\mathrm{pH}$ environment which occurs during regular HD even for a short time may lead to vascular calcification [94]. Thiosulfate, previously taken for urolithiasis events, nowadays seem to reduce vascular calcification [95]. Additional clinical trials on Thiosulfate are still underway [96]. Vitamin K analogues serve as cofactors for gamma-carboxylation of proteins including coagulation factors and matrix gla protein (MGP). MGP regulate vascular calcification, and its deficiency may lead to severe VC and CVD [50].

\section{Conclusion}

Vascular calcification started with extracellular phosphate regulation of vascular smooth muscle cell osteogenic differentiation and calcium phosphate mineralization. Generalized inflammatory condition in ESRD patients also suggested playing as an important factor in VC. FGF23-Klotho axis in regulating serum $\mathrm{P}$ levels and their roles in CVD become uncovered recently, while newer concepts of PTH and vitamin $\mathrm{D}$ are still evolving. It is increasingly accepted that factors promoting VC and inhibiting VC are in balance with excess of any one may relate with adverse effects in CKD$\mathrm{MBD}$ and VC. Management trends are shifting from treating $\mathrm{VC}$ in older days to preventing VC in the new era. For the last, but not the least, the main target for all those efforts is to improve survival and life quality of these ESRD patients.

\section{Authors' Contributions}

C.-M. Z. and K.-C. L. contributed equally to this work.

\section{References}

[1] J. L. Reynolds, A. J. Joannides, J. N. Skepper et al., "Human vascular smooth muscle cells undergo vesicle-mediated calcification in response to changes in extracellular calcium and phosphate concentrations: a potential mechanism for accelerated vascular calcification in ESRD," Journal of the American Society of Nephrology, vol. 15, no. 11, pp. 2857-2867, 2004.

[2] S. Hara and K. Yokoyama, "Mechanism and therapy of vascular calcification," Clinical Calcium, vol. 14, no. 5, pp. 752-755, 2004.

[3] G. A. Block, T. E. Hulbert-Shearon, N. W. Levin, and F. K. Port, "Association of serum phosphorus and calcium x phosphate product with mortality risk in chronic hemodialysis patients: a national study," American Journal of Kidney Diseases, vol. 31, no. 4, pp. 607-617, 1998.

[4] G. A. Block, P. S. Klassen, J. M. Lazarus, N. Ofsthun, E. G. Lowrie, and G. M. Chertow, "Mineral metabolism, mortality, and morbidity in maintenance hemodialysis," Journal of the American Society of Nephrology, vol. 15, no. 8, pp. 2208-2218, 2004.

[5] M. Noordzij, E. M. Cranenburg, L. F. Engelsman et al., "Progression of aortic calcification is associated with disorders of mineral metabolism and mortality in chronic dialysis patients," Nephrology Dialysis Transplantation, vol. 26, no. 5, pp. 1662-1669, 2011.

[6] C. P. Kovesdy, "How KDIGO will (or will not) influence the management of hyperphosphatemia," Semin Dial, vol. 24, no. 1, pp. 35-36, 2011.

[7] K. J. Martin and E. A. Gonzalez, "Prevention and control of phosphate retention/hyperphosphatemia in CKD-MBD: what is normal, when to start, and how to treat?" Clinical Journal of the American Society of Nephrology, vol. 6, no. 2, pp. 440-446, 2011. 
[8] A. P. Sage, J. Lu, Y. Tintut, and L. L. Demer, "Hyperphosphatemia-induced nanocrystals upregulate the expression of bone morphogenetic protein-2 and osteopontin genes in mouse smooth muscle cells in vitro," Kidney International, vol. 79, no. 4, pp. 414-422, 2011.

[9] K. A. Hruska, S. Mathew, R. Lund, P. Qiu, and R. Pratt, "Hyperphosphatemia of chronic kidney disease," Kidney International, vol. 74, no. 2, pp. 148-157, 2008.

[10] V. Savica, L. A. Calò, A. Granata et al., "A new approach to the evaluation of hyperphosphatemia in chronic kidney disease," Clinical Nephrology, vol. 68, no. 4, pp. 216-221, 2007.

[11] T. Maruyama and D. Kadowaki, "To facilitate the compliance of phosphate binder for the control of hyperphosphatemia in chronic kidney disease patients," Clinical Calcium, vol. 19, no. 2, pp. 248-252, 2009.

[12] M. Abedin, Y. Tintut, and L. L. Demer, "Vascular calcification: mechanisms and clinical ramifications," Arteriosclerosis, Thrombosis, and Vascular Biology, vol. 24, no. 7, pp. 11611170, 2004.

[13] J. Oh, R. Wunsch, M. Turzer et al., "Advanced coronary and carotid arteriopathy in young adults with childhood-onset chronic renal failure," Circulation, vol. 106, no. 1, pp. 100-105, 2002.

[14] A. Farzaneh-Far and K. Amann, "Morphology of coronary atherosclerotic lesions in patients with end-stage renal failure (multiple letters) [4]," Nephrology Dialysis Transplantation, vol. 15, no. 10, p. 1710, 2000.

[15] U. Schwarz, M. Buzello, E. Ritz et al., "Morphology of coronary atherosclerotic lesions in patients with end-stage renal failure," Nephrology Dialysis Transplantation, vol. 15, no. 2, pp. 218-223, 2000.

[16] M. Yuce, V. Davutoglu, and M. Cakici, "Fetuin-A, osteoporosis and inflammation-proposal of possible mechanisms for vascular and valvular calcification in chronic kidney disease," Nephrology Dialysis Transplantation, vol. 25, no. 8, p. 2801, 2010.

[17] B. Kestenbaum, J. N. Sampson, K. D. Rudser et al., "Serum phosphate levels and mortality risk among people with chronic kidney disease," Journal of the American Society of Nephrology, vol. 16, no. 2, pp. 520-528, 2005.

[18] S. J. Marchais, A. P. Guerin, and G. M. London, "Arterial calcinosis, chronic renal failure and calcium antagonism," Drugs, vol. 44, supplement 1, pp. 119-122, 1992.

[19] A. P. Guérin, G. M. London, S. J. Marchais, and F. Metivier, "Arterial stiffening and vascular calcifications in end-stage renal disease," Nephrology Dialysis Transplantation, vol. 15, no. 7, pp. 1014-1021, 2000.

[20] J. R. Wu-Wong, M. Nakane, J. Ma, X. Ruan, and P. E. Kroeger, "Elevated phosphorus modulates vitamin D receptor-mediated gene expression in human vascular smooth muscle cells," American Journal of Physiology, vol. 293, no. 5, pp. F1592-F1604, 2007.

[21] A. Di Benedetto, D. Marcelli, A. D’Andrea et al., "Risk factors and underlying cardiovascular diseases in incident ESRD patients," Journal of Nephrology, vol. 18, no. 5, pp. 592-598, 2005.

[22] M. Tonelli, F. Sacks, M. Pfeffer, Z. Gao, and G. Curhan, "Relation between serum phosphate level and cardiovascular event rate in people with coronary disease," Circulation, vol. 112, no. 17, pp. 2627-2633, 2005.

[23] E. Ishimura, S. Okuno, H. Taniwaki et al., "Different risk factors for vascular calcification in end-stage renal disease between diabetics and nondiabetics: the respective importance of glycemic and phosphate control," Kidney and Blood Pressure Research, vol. 31, no. 1, pp. 10-15, 2008.

[24] E. Neven and P. C. D'Haese, "Vascular calcification in chronic renal failure: what have we learned from animal studies?" Circulation Research, vol. 108, no. 2, pp. 249-264, 2011.

[25] N. F. Goodman, M. B. Bledsoe, W. Futterweit et al., "American Association of Clinical Endocrinologists medical guidelines for the clinical practice for the diagnosis and treatment of hyperandrogenic disorders," Endocrine Practice, vol. 7, no. 2, pp. 120-134, 2001.

[26] I. Bhan and R. Thadhani, "Vascular calcification and ESRD: a hard target," Clinical Journal of the American Society of Nephrology, vol. 4, no. 1, pp. S102-S105, 2009.

[27] B. R. Di Iorio, S. Bortone, C. Piscopo et al., "Cardiac vascular calcification and QT interval in ESRD patients: is there a link?" Blood Purification, vol. 24, no. 5-6, pp. 451-459, 2006.

[28] D. A. Towler, "Vascular calcification in ESRD: another cloud appears in the perfect storm-but highlights a silver lining?" Kidney International, vol. 66, no. 6, pp. 2467-2468, 2004.

[29] S. J. Silverberg, E. Shane, T. L. Clemens et al., "The effect of oral phosphate administration on major indices of skeletal metabolism in normal subjects," Journal of Bone and Mineral Research, vol. 1, no. 4, pp. 383-388, 1986.

[30] K. C. Lu, C. F. Tseng, C. C. Wu et al., "Effects of calcitriol on type $5 b$ tartrate-resistant acid phosphatase and interleukin6 in secondary hyperparathyroidism," Blood Purification, vol. 24, no. 5-6, pp. 423-430, 2006.

[31] C. -C. Wu, J. -H. Chang, C. -C. Chen et al., "Calcitriol treatment attenuates inflammation and oxidative stress in hemodialysis patients with secondary hyperparathyroidism," Tohoku Journal of Experimental Medicine, vol. 223, no. 3, pp. 153-159, 2011.

[32] A. A. Portale, B. P. Halloran, M. M. Murphy, and R. C. Morris, "Oral intake of phosphorus can determine the serum concentration of 1,25-dihydroxyvitamin $\mathrm{D}$ by determining its production rate in humans," Journal of Clinical Investigation, vol. 77, no. 1, pp. 7-12, 1986.

[33] A. A. Portale, B. P. Halloran, and R. C. Morris Jr., "Physiologic regulation of the serum concentration of 1,25dihydroxyvitamin D by phosphorus in normal men," Journal of Clinical Investigation, vol. 83, no. 5, pp. 1494-1499, 1989.

[34] A. J. Bleyer, J. Burkart, M. Piazza, G. Russell, M. Rohr, and J. J. Carr, "Changes in cardiovascular calcification after parathyroidectomy in patients with ESRD," American Journal of Kidney Diseases, vol. 46, no. 3, pp. 464-469, 2005.

[35] T. Stompór, M. Pasowicz, W. Sułowicz et al., "An association between coronary artery calcification score, lipid profile, and selected markers of chronic inflammation in ESRD patients treated with peritoneal dialysis," American Journal of Kidney Diseases, vol. 41, no. 1, pp. 203-211, 2003.

[36] G. Schlieper, R. Westenfeld, V. Brandenburg, and M. Ketteler, "Inhibitors of calcification in blood and urine," Seminars in Dialysis, vol. 20, no. 2, pp. 113-121, 2007.

[37] H. H. T. Hsu and B. G. Abbo, "Role of bicarbonate/CO2 buffer in the initiation of vesicle-mediated calcification: mechanisms of aortic calcification related to atherosclerosis," Biochimica et Biophysica Acta, vol. 1690, no. 2, pp. 118-123, 2004.

[38] A. Shioi, "Molecular mechanisms of vascular calcification," Clinical Calcium, vol. 20, no. 11, pp. 1611-1619, 2010.

[39] A. P. Sage, Y. Tintut, and L. L. Demer, "Regulatory mechanisms in vascular calcification," Nature Reviews Cardiology, vol. 7, no. 9, pp. 528-536, 2010. 
[40] M. C. Yadav, A. M. Simão, S. Narisawa et al., "Loss of skeletal mineralization by the simultaneous ablation of $\mathrm{PHOSPHO} 1$ and alkaline phosphatase function: a unified model of the mechanisms of initiation of skeletal calcification," Journal of Bone and Mineral Research, vol. 26, no. 2, pp. 286-297, 2011.

[41] R. G. Hammonds Jr., R. Schwall, A. Dudley et al., "Boneinducing activity of mature BMP-2b produced from a hybrid BMP-2a/2b precursor," Molecular Endocrinology, vol. 5, no. 1, pp. 149-155, 1991.

[42] K. V. Brown, B. Li, T. Guda, D. S. Perrien, S. A. Guelcher, and J. C. Wenke, "Improving bone formation in a rat femur segmental defect by controlling BMP-2 release," Tissue Engineering Part $A$. In press.

[43] H. Yu, P. de Vos, and Y. Ren, "Overexpression of osteoprotegerin promotes preosteoblast differentiation to mature osteoblasts," Angle Orthodontist, vol. 81, no. 1, pp. 100-106, 2011.

[44] M. Waluś-Miarka, B. Katra, D. Fedak et al., "Osteoprotegerin is associated with markers of atherosclerosis and body fat mass in type 2 diabetes patients," International Journal of Cardiology, vol. 147, no. 2, pp. 335-336, 2011.

[45] D. A. Prosdocimo, S. C. Wyler, A. M. Romani, W. C. O’Neill, and G. R. Dubyak, "Regulation of vascular smooth muscle cell calcification by extracellular pyrophosphate homeostasis: synergistic modulation by cyclic AMP and hyperphosphatemia," American Journal of Physiology, vol. 298, no. 3, pp. C702-C713, 2010.

[46] Y. Jiang and M. Wang, "Overexpression of parathyroid pituitary-specific transcription factor (Pit)-1 in hyperphosphatemia-induced hyperparathyroidism of chronic renal failure rats," Chinese Medical Journal (English Edition), vol. 123, no. 12, pp. 1566-1570, 2010.

[47] A. Sugita, S. Kawai, T. Hayashibara et al., "Cellular ATP synthesis mediated by type III sodium-dependent phosphate transporter Pit-1 is critical to chondrogenesis," The Journal of Biological Chemistry, vol. 286, no. 4, pp. 3094-3103, 2011.

[48] C. M. Giachelli, "Vascular calcification mechanisms," Journal of the American Society of Nephrology, vol. 15, no. 12, pp. 2959 2964, 2004.

[49] R. Villa-Bellosta, A. Millan, and V. Sorribas, "Role of calciumphosphate deposition in vascular smooth muscle cell calcification," American Journal of Physiology, vol. 300, no. 1, pp. C210C220, 2011.

[50] G. Luo, P. Ducy, M. D. McKee et al., "Spontaneous calcification of arteries and cartilage in mice lacking matrix GLA protien," Nature, vol. 386, no. 6620, pp. 78-81, 1997.

[51] G. Molostvov, S. Fletcher, R. Bland, and D. Zehnder, "Extracellular calcium-sensing receptor mediated signalling is involved in human vascular smooth muscle cell proliferation and apoptosis," Cellular Physiology and Biochemistry, vol. 22, no. 5-6, pp. 413-422, 2008.

[52] C. Boudot, Z. Saidak, A. K. Boulanouar et al., "Implication of the calcium sensing receptor and the Phosphoinositide 3-kinase/Akt pathway in the extracellular calcium-mediated migration of RAW 264.7 osteoclast precursor cells," Bone, vol. 46, no. 5, pp. 1416-1423, 2010.

[53] M. Rodríguez, E. Aguilera-Tejero, F. J. Mendoza, F. Guerrero, and I. López, "Effects of calcimimetics on extraskeletal calcifications in chronic kidney disease," Kidney International, no. 111, pp. S50-S54, 2008.

[54] A. Caudrillier, R. Mentaverri, M. Brazier, S. Kamel, and Z. A. Massy, "Calcium-sensing receptor as a potential modulator of vascular calcification in chronic kidney disease," Journal of Nephrology, vol. 23, no. 1, pp. 17-22, 2010.
[55] F. Tentori, J. M. Albert, E. W. Young et al., "The survival advantage for haemodialysis patients taking vitamin $\mathrm{D}$ is questioned: findings from the Dialysis Outcomes and Practice Patterns Study," Nephrology Dialysis Transplantation, vol. 24, no. 3, pp. 963-972, 2009.

[56] M. M. Zhao, M. J. Xu, Y. Cai et al., "Mitochondrial reactive oxygen species promote p65 nuclear translocation mediating high-phosphate-induced vascular calcification in vitro and in vivo," Kidney International, vol. 79, no. 10, pp. 1071-1079, 2011.

[57] J. D. McCanless, J. A. Cole, S. M. Slack, J. D. Bumgardner, P. O. Zamora, and W. O. Haggard, "Modeling nucleus pulposus regeneration in vitro: mesenchymal stem cells, alginate beads, hypoxia, BMP-2, and synthetic peptide B2A," Spine (Phila Pa 1976). In press.

[58] H. Juppner, "Phosphate and FGF-23," Kidney International, vol. 121, pp. S24-S27, 2011.

[59] D. Fliser, B. Kollerits, U. Neyer et al., "Fibroblast growth factor 23 (FGF23) predicts progression of chronic kidney disease: the Mild to Moderate Kidney Disease (MMKD) study," Journal of the American Society of Nephrology, vol. 18, no. 9, pp. 26012608, 2007.

[60] M. M. Nasrallah, A. R. El-Shehaby, M. M. Salem, N. A. Osman, E. El Sheikh, and U. A. Sharaf El Din, "Fibroblast growth factor-23 (FGF-23) is independently correlated to aortic calcification in haemodialysis patients," Nephrology, Dialysis, Transplantation, vol. 25, no. 8, pp. 2679-2685, 2010.

[61] D. Yonova, "Vascular calcification and metabolic acidosis in end stage renal disease," Hippokratia, vol. 13, no. 3, pp. 139140, 2009.

[62] Z. Al-Aly, "Metabolic acidosis and vascular calcification: using blueprints from bone to map a new venue for vascular research," Kidney International, vol. 73, no. 4, pp. 377-379, 2008.

[63] P. Chu, K. C. Lu, and Y. F. Lin, "Acute correction of metabolic acidosis increases serum procollagen type I carboxyterminal propeptide in patients with chronic renal failure," Journal of the Formosan Medical Association, vol. 100, no. 11, pp. 748752, 2001.

[64] K. C. Lu, S. H. Lin, F. C. Yu, S. H. Chyr, and S. D. Shieh, "Influence of metabolic acidosis on serum 1,25(OH)D levels in chronic renal failure," Mineral and Electrolyte Metabolism, vol. 21, no. 6, pp. 398-402, 1995.

[65] Y. F. Lin, S. D. Shieh, L. K. Diang et al., "Influence of rapid correction of metabolic acidosis on serum osteocalcin level in chronic renal failure," ASAIO Journal, vol. 40, no. 3, pp. M440M444, 1994

[66] F. J. Mendoza, I. Lopez, A. Montes De Oca, J. Perez, M. Rodriguez, and E. Aguilera-Tejero, "Metabolic acidosis inhibits soft tissue calcification in uremic rats," Kidney International, vol. 73, no. 4, pp. 407-414, 2008.

[67] J. Y. Wang, K. C. Lu, Y. F. Lin, and W. M. Hu, "Correlation of serum leptin concentrations with body composition and gender in taiwanese hemodialysis patients without diabetes," Renal Failure, vol. 25, no. 6, pp. 953-966, 2003.

[68] M. Zeadin, M. Butcher, G. Werstuck, M. Khan, C. K. Yee, and S. G. Shaughnessy, "Effect of leptin on vascular calcification in apolipoprotein E-deficient mice," Arteriosclerosis, Thrombosis, and Vascular Biology, vol. 29, no. 12, pp. 2069-2075, 2009.

[69] S. S. Martin, A. Qasim, and M. P. Reilly, "Leptin Resistance. A possible interface of inflammation and metabolism in obesityrelated cardiovascular disease," Journal of the American College of Cardiology, vol. 52, no. 15, pp. 1201-1210, 2008. 
[70] V. Savica, L. A. Calò, P. Monardo et al., "Salivary phosphatebinding chewing gum reduces hyperphosphatemia in dialysis patients," Journal of the American Society of Nephrology, vol. 20, no. 3, pp. 639-644, 2009.

[71] V. P. Persy and M. D. McKee, "Prevention of vascular calcification: is pyrophosphate therapy a solution?" Kidney International, vol. 79, no. 5, pp. 490-493, 2011.

[72] K. A. Lomashvili, W. Khawandi, and W. C. O’Neill, "Reduced plasma pyrophosphate levels in hemodialysis patients," Journal of the American Society of Nephrology, vol. 16, no. 8, pp. 24952500, 2005.

[73] W. C. O’Neill, K. A. Lomashvili, H. H. Malluche, M.-C. Faugere, and B. L. Riser, "Treatment with pyrophosphate inhibits uremic vascular calcification," Kidney International, vol. 79, no. 5, pp. 512-517, 2011.

[74] H. Segawa, I. Kaneko, S. Yamanaka et al., "Intestinal Na-P cotransporter adaptation to dietary $\mathrm{P}(\mathrm{i})$ content in vitamin $\mathrm{D}$ receptor null mice," American Journal of Physiology, vol. 287, no. 1, pp. F39-F47, 2004.

[75] G. M. Chertow, S. K. Burke, and P. Raggi, "Sevelamer attenuates the progression of coronary and aortic calcification in hemodialysis patients," Kidney International, vol. 62, no. 1, pp. 245-252, 2002.

[76] T. Kakuta, R. Tanaka, T. Hyodo et al., "Effect of sevelamer and calcium-based phosphate binders on coronary artery calcification and accumulation of circulating advanced glycation end products in hemodialysis patients," American Journal of Kidney Diseases, vol. 57, no. 3, pp. 422-431, 2011.

[77] G. A. Block, D. M. Spiegel, J. Ehrlich et al., "Effects of sevelamer and calcium on coronary artery calcification in patients new to hemodialysis," Kidney International, vol. 68, no. 4, pp. 1815-1824, 2005.

[78] T. H. Schindler, J. Cadenas, A. D. Facta et al., "Improvement in coronary endothelial function is independently associated with a slowed progression of coronary artery calcification in type 2 diabetes mellitus," European Heart Journal, vol. 30, no. 24, pp. 3064-3073, 2009.

[79] S. Damment, R. Secker, V. Shen, V. Lorenzo, and M. Rodriguez, "Long-term treatment with lanthanum carbonate reduces mineral and bone abnormalities in rats with chronic renal failure," Nephrology Dialysis Transplantation. In press.

[80] L. Noto, "Lanthanum carbonate provides control of phosphorus levels in patients new to phosphate binder therapy and patients changed from other phosphate binders," Journal of Renal Nutrition, vol. 21, no. 3, pp. 277-282, 2011.

[81] V. M. Brandenburg, W. Jahnen-Dechent, and M. Ketteler, "Sevelamer and the bone-vascular axis in chronic kidney disease: bone turnover, inflammation, and calcification regulation," Kidney International, no. 114, pp. S26-33, 2009.

[82] J. M. Frazão and T. Adragão, "Treatment of hyperphosphatemia with sevelamer hydrochloride in dialysis patients: effects on vascular calcification, bone and a close look into the survival data," Kidney International, no. 111, pp. S38-S43, 2008.

[83] S. Mathew, R. J. Lund, F. Strebeck, K. S. Tustison, T. Geurs, and K. A. Hruska, "Reversal of the adynamic bone disorder and decreased vascular calcification in chronic kidney disease by sevelamer carbonate therapy," Journal of the American Society of Nephrology, vol. 18, no. 1, pp. 122-130, 2007.

[84] G. A. Block, S. L. Brillhart, M. S. Persky, A. Amer, and A. J. Slade, "Efficacy and safety of SBR759, a new iron-based phosphate binder," Kidney International, vol. 77, no. 10, pp. 897-903, 2010.
[85] W. Waas and W. Hasselbach, "Interference of nucleoside diphosphates and inorganic phosphate with nucleosidetriphosphate-dependent calcium fluxes and calciumdependent nucleoside-triphosphate hydrolysis in membranes of sarcoplasmic-reticulum vesicles," European Journal of Biochemistry, vol. 116, no. 3, pp. 601-608, 1981.

[86] M. D. Francis, R. G. G. Russell, and H. Fleisch, "Diphosphonates inhibit formation of calcium phosphate crystals in vitro and pathological calcification in vivo," Science, vol. 165, no. 3899, pp. 1264-1266, 1969.

[87] J. L. Meyer, "Can biological calcification occur in the presence of pyrophosphate?" Archives of Biochemistry and Biophysics, vol. 231, no. 1, pp. 1-8, 1984.

[88] C. E. Fiore, P. Pennisi, I. Pulvirenti, and C. M. Francucci, "Bisphosphonates and atherosclerosis," Journal of Endocrinological Investigation, vol. 32, no. 4, pp. 38-43, 2009.

[89] Y. S. Silay, K. Altundag, O. Altundag, M. A. Atik, and M. Ozen, "Bisphosphonates may inhibit development of atherosclerosis formation through its bactericidal effect on nanobacteria," Medical Hypotheses, vol. 64, no. 6, pp. 1239-1240, 2005.

[90] S. Elmariah, J. A. Delaney, K. D. O’Brien et al., “Bisphosphonate use and prevalence of valvular and vascular calcification in women MESA (The Multi-Ethnic Study of Atherosclerosis)," Journal of the American College of Cardiology, vol. 56, no. 21, pp. 1752-1759, 2010.

[91] D. Müller, H. Mehling, B. Otto et al., "Niacin lowers serum phosphate and increases HDL cholesterol in dialysis patients," Clinical Journal of the American Society of Nephrology, vol. 2, no. 6, pp. 1249-1254, 2007.

[92] D. MacCubbin, D. Tipping, O. Kuznetsova, W. A. Hanlon, and A. G. Bostom, "Hypophosphatemic effect of niacin in patients without renal failure: a randomized trial," Clinical Journal of the American Society of Nephrology, vol. 5, no. 4, pp. 582-589, 2010.

[93] V. Savica, L. A. Calò, P. Monardo et al., "Salivary phosphatebinding chewing gum reduces hyperphosphatemia in dialysis patients," Journal of the American Society of Nephrology, vol. 20, no. 3, pp. 639-644, 2009.

[94] K. Lomashvili, P. Garg, and W. C. O’Neill, "Chemical and hormonal determinants of vascular calcification in vitro," Kidney International, vol. 69, no. 8, pp. 1464-1470, 2006.

[95] M. Kalisiak, M. Courtney, A. Lin, and A. Brassard, "Calcific uremic arteriolopathy (calciphylaxis): successful treatment with sodium thiosulfate in spite of elevated serum phosphate," Journal of Cutaneous Medicine and Surgery, vol. 13, supplement 1, pp. S29-S34, 2009.

[96] J. S. Cicone, J. B. Petronis, C. D. Embert, and D. A. Spector, "Successful treatment of calciphylaxis with intravenous sodium thiosulfate," American Journal of Kidney Diseases, vol. 43, no. 6, pp. 1104-1108, 2004. 


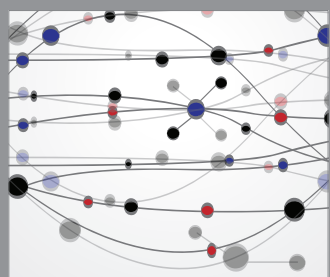

The Scientific World Journal
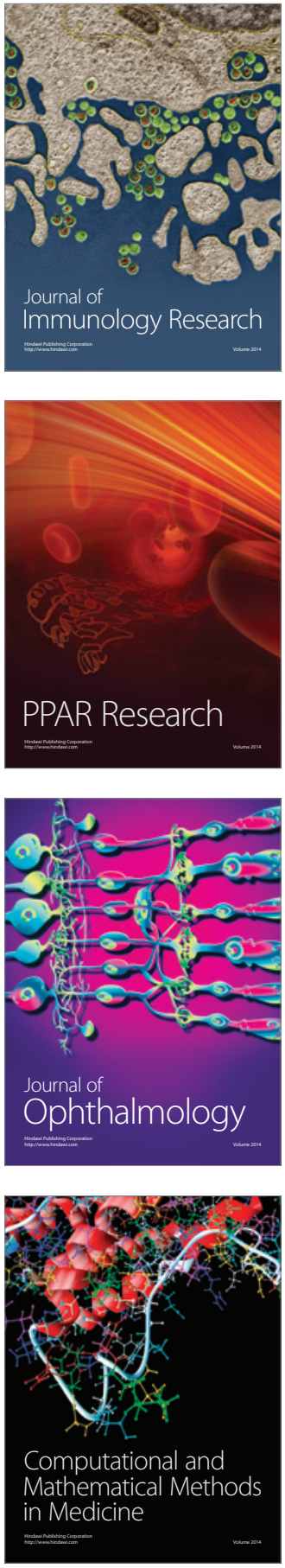

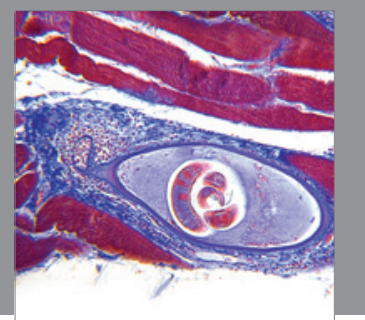

Gastroenterology

Research and Practice
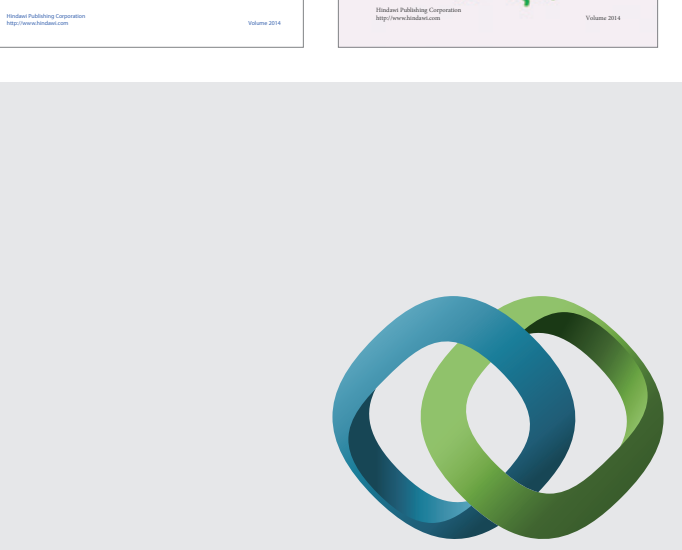

\section{Hindawi}

Submit your manuscripts at

http://www.hindawi.com
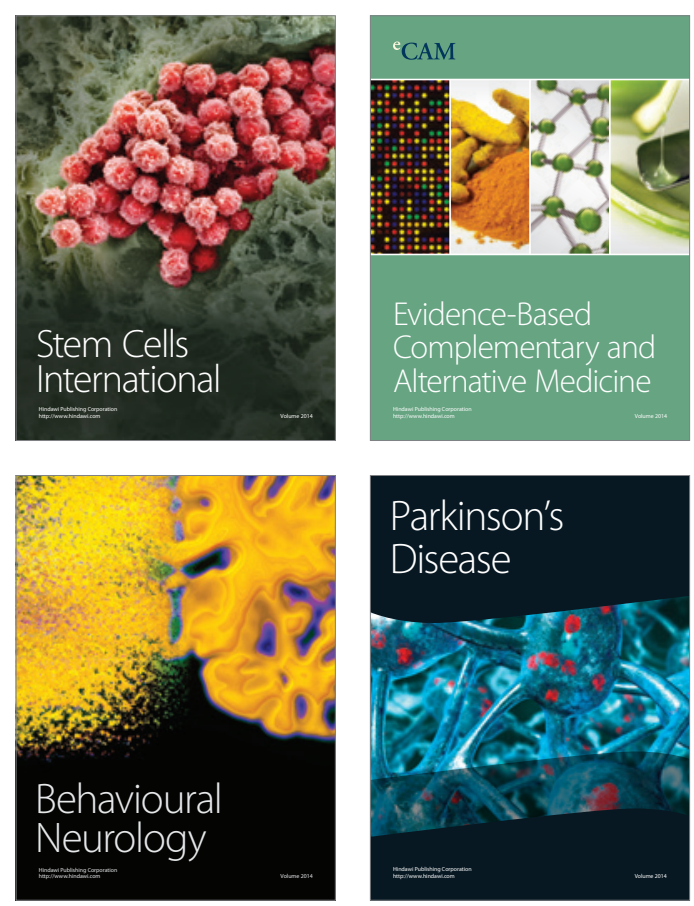

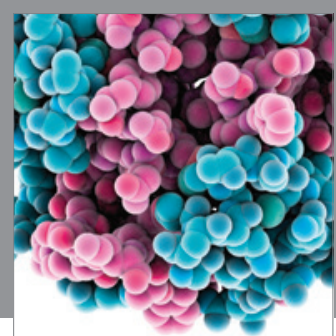

Journal of
Diabetes Research

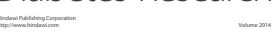

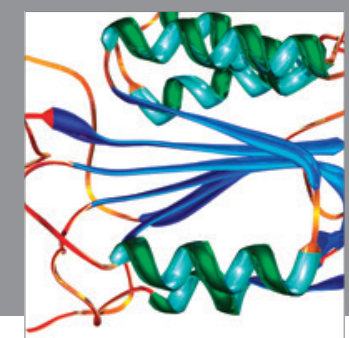

Disease Markers
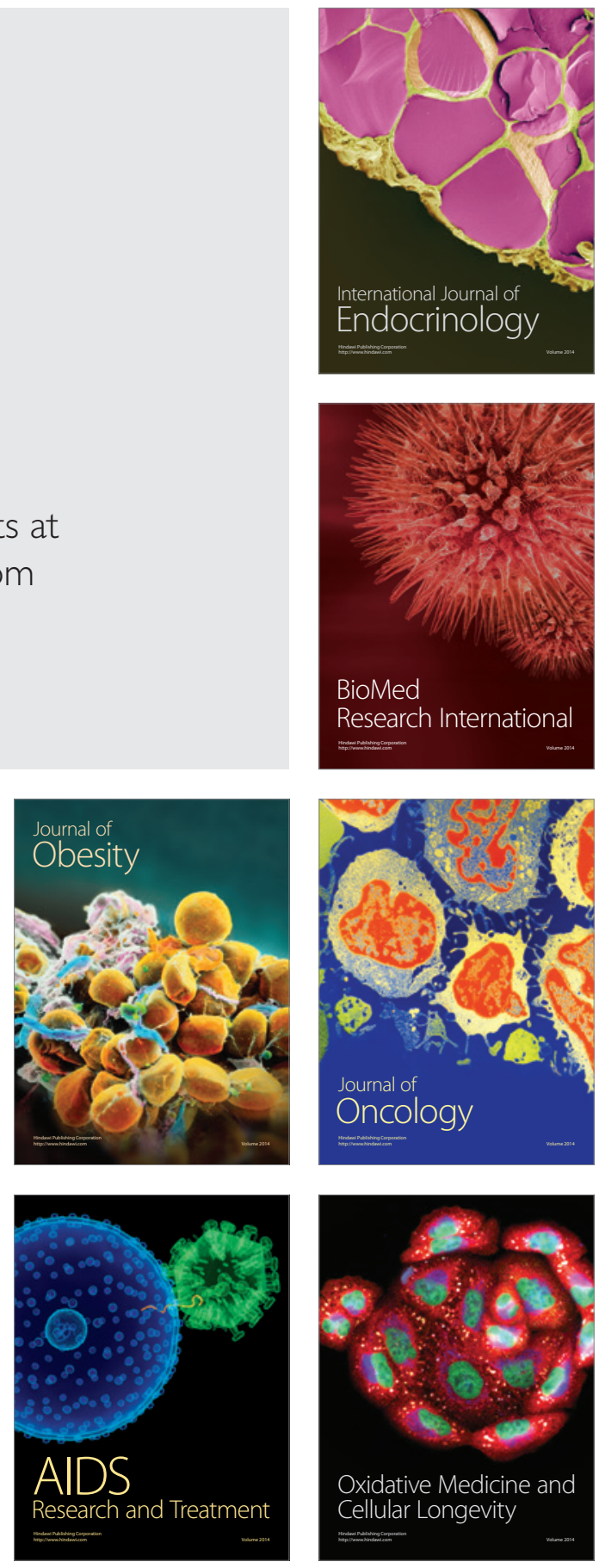